\title{
Semiautomatic Volume Conductor Modeling Pipeline for Imaging the Cardiac Electrophysiology Noninvasively
}

\author{
Bernhard Pfeifer ${ }^{1}$, Michael Seger ${ }^{1}$, Christoph Hintermüller ${ }^{1}$, Gerald Fischer ${ }^{1}$, \\ Friedrich Hanser ${ }^{1}$, Robert Modre ${ }^{2}$, Hannes Mühlthaler ${ }^{3}$, and Bernhard Tilg ${ }^{1}$ \\ ${ }^{1}$ Institute of Biomedical Engineering, University for Health sciences, medical \\ informatics and technology, Austria \\ bernhard.pfeifer@umit.at \\ 2 ARC Seibersdorf research GmbH, Austria \\ ${ }^{3}$ Department of Vascular Surgery, Innsbruck Medical University, Innsbruck
}

\begin{abstract}
In this paper we present an approach for extracting patient individual volume conductor models (VCM) using volume data acquired from Magnetic Resonance Imaging (MRI) for computational biology of electrical excitation in the patient's heart. The VCM consists of the compartments chest surface, lung surfaces, the atrial and ventricular myocardium, and the blood masses. For each compartment a segmentation approach with no or little necessity of user interaction was implemented and integrated into a VCM segmentation pipeline to enable the inverse problem of electrocardiography to become clinical applicable. The segmentation pipeline was tested using volume data from ten patients with structurally normal hearts.
\end{abstract}

\section{Introduction}

Computer modeling of bioelectric activity is of interest in cardiac electrophysiology [12] and, furthermore, the noninvasive imaging of the electrical excitation from electrocardiogram (ECG) becomes more and more a diagnosis tool in clinical application 34]. For enabling noninvasive imaging four dimensional (3D and time) anatomical and ECG mapping data have to be combined. The anatomical data are obtained via MRI, and are the basis for generating a VCM. A VCM for solving the inverse problem consists of the electrostatically most relevant compartments, which are chest surface, lung surface, the atrial and ventricular myocardium and the cardiac blood masses. The ECG mapping data are acquired with a multi-channel ECG recording system [56].

In clinical practice the localization of ectopic origins is currently achieved by traditional catheter techniques and by recently introduced catheter mapping systems, like the CARTO ${ }^{\mathrm{TM}}$ system [7. The major advantage of the CARTO $^{\mathrm{TM}}$ system is that it enables the combination between electrical and spatial endocardial information, which supports the cardiologist in getting a deeper insight in the patients individual arrhythmia. $\mathrm{CARTO}^{\mathrm{TM}}$ provides anatomical information that 
correlates to the electrogram mapping and the tagging system assists the creation of a linear lesion. CARTO ${ }^{\mathrm{TM}}$ shows significant limitations when it comes to acquiring single-beat activation maps and, furthermore, these are mostly applied to single target chambers only. Another important fact is that it is impossible to obtain information from the epicardium under clinical conditions. Moreover, this technique is invasive which involves a risk to the patient during the procedure. These limitations are repealed and the information about the electrical excitation can be provided noninvasively, when applying the noninvasive imaging of cardiac electrophysiology (NICE) approach, provided that a VCM exists.

\section{Implementation}

For the semi-automatic segmentation of a VCM a framework has been developed using Java. The reason choosing Java was, that we have been developing web services for VCM modeling. A web service is a piece of logic that is placed on a server anyplace and can be operated using standard protocols like HTTP. The web service enables the functionality to handle distributed data, that exists when dealing with medical images in clinical settings, and allows to couple different services in order to generate new ones. Another important fact that argues for the usage of this technique is that the user only needs to have a Thin Client (usually an Internet Browser) installed, which should be standard on nearly every computer system, and the different time-consuming segmentation tasks can be executed parallel on different dedicated computing systems or a computing cluster.

The environment for executing the electrical field computation and for visualization of the models and results is amiraDev ${ }^{\mathrm{TM}} 3.0$ (TGS Europe Inc.). The amiraDev $^{\mathrm{TM}}$ environment is a visualization tool, which gives the opportunity to expand the functionality by implementing dynamic link libraries using the amiraDev $^{\mathrm{TM}}$ library.

\section{Methods}

\subsection{Model Properties}

When a VCM is used for imaging the electrical excitation in the heart a functional model instead of a detailed anatomical model is needed. The difference between an anatomical model and the desired functional VCM can be explained by the smoothing properties of the Fredholm integral, which describes the relationship between the source in the heart (transmembrane potential (TMP) distribution) and the potentials on the chest surface. Therefore, fine anatomical structures do not influence the macroscopic electrical properties, and can be smoothed out without decreasing the model's accuracy.

For the lung and chest extraction a T1 flash, non contrasted axial volume data set with a slice thickness of $10 \mathrm{~mm}$ is taken. For the cardiac model, a cinegated, T1-flash, non contrasted short-axis volume data set with a slice thickness of $6 \mathrm{~mm}$ for the ventricles and $4 \mathrm{~mm}$ for the atria is used. 


\subsection{Volume Conductor Model Extraction}

The VCM used for estimating the electrical excitation in the patient individual heart consists of the compartments chest, lungs, atrial and ventricular myocardium, and the blood masses. A detailed explanation of the electrostatically most relevant compartments can be found in 8 . Because of the generally unknown individual fiber orientation, electrical isotropy is assumed in the surface heart model approach based on the bidomain theory.

Chest Extraction: The volume data are prefiltered using a Roberts Cross filter function [9], which highlights regions of high spatial gradient measurements which corresponds to edges. In the next step the minimal spanning bounding box (region of interest (ROI)) of the chest in the volume data is determined automatically. From the left and right upper voxel of the ROI a virtual beam with a 45 degree angle is projected to the chests border, which marks the border of the chest and tries to exclude the arms at those positions where they do not longer touch the body in order to get the torso.

Lung Extraction: The big advantage when trying to segment the lungs is, that the gray-values of the lungs differ intensely from the surrounding tissues. The main problem is, that the left and the right lungs are often in immediate contact, but for our electrical excitation estimation we need the lungs as separate compartments, due to implementation details of our inverse solver. Briefly the segmentation task can be described following way: first the volume data is thresholded to separate lungs from the surrounding fat, then the chest surrounding air, noise and airways are removed, and by usage of morphological operators the lung borders are rebuilt. In a last step the lungs are separated by a connected component labeling approach. More information about this approach can be found at 10 .

Cardiac Blood Mass Extraction: Active Appearance Models (AAM) [1] have been shown to be useful for interpreting deformable objects. They contain statistical models of shape and gray-level appearance (texture) of objects and can be used for searching the desired structure in unseen images. The segmentation procedure is processed by synthesizing objects and by minimizing the error between the target and the synthesized object.

Objects in images are represented using shapes, which can be described by a set of $n$ points. Shapes are placed in the training images by searching for corresponding landmark points, which was in our case done manually. A vector representing a shape can be defined as:

$$
s=\left(x_{1}, \ldots, x_{n}, y_{1}, \ldots, y_{n}\right)^{T} .
$$

After having placed the landmark points of the different training images the shapes have to be realigned. Effects of scaling, translation and rotation have to be removed to enable statistics. All shapes are aligned in a way, that the sum of squared distances of each shape to the mean shape $\left(D=\sum\left|s_{i}-\bar{s}\right|^{2}\right)$ is 
minimized. As mentioned before, an AAM represents shape and texture changes learnt during the training. The shape can be represented as a vector $s$ and the texture as a vector $g$ :

$$
\begin{aligned}
& s=\bar{s}+Q_{s} c, \\
& g=\bar{g}+Q_{g} c,
\end{aligned}
$$

where $\bar{s}$ represents the mean shape, $\bar{g}$ represents the mean texture, $Q_{s}$ and $Q_{g}$ are describing the modes of variation and $c$ is the parameter vector controlling the shape and texture.

When using an AAM for segmentation the model seeks to minimize the difference between unseen image and one created by the appearance model. A difference vector $\delta I$ can be defined as

$$
\delta I=I_{i}-I_{m},
$$

where $I_{i}$ describes the texture of the image and $I_{m}$ the texture of the current model. The best match can be obtained when minimizing the magnitude of the squared difference vector:

$$
\Delta=|\delta I|^{2} .
$$

More information can be found in 12 .

Ventricular Blood Mass Model: The models are generated using volume data from eight different cine-gated short-axis cardiac MRI scans. From each individual volume stack 15 images containing the ventricular blood masses at 4 different trigger time stages were taken. The landmarks were placed manually. For the left and the right ventricular blood mass boundary 33 landmarks were used, and more 33 landmark points were used for marking the outer boundary of the left ventricular myocardium.

Atrial Blood Mass Model: Due to the more complex geometry and, therefore, the more distinctive shape variations, the AAM model building approach needs to be more specific. To handle this task so called divisions of appearance are introduced. For the atrial blood mass extraction at least three divisions are necessary in order to cover the shape variations of the atria. The first division reaches from the base of the heart up to the left upper and the left lower pulmonary vein. The second division reaches from there up to the last appearance of the left upper and left lower pulmonary vein. From this position up to the right lower and right upper pulmonary vein the third division completes the overall AAM model. The same volume data of the eight patients was used to create the models. For each division 5 images at 4 different trigger states were taken. The mean Dice similarity coefficient (DSC) between the images taken for division one is 0.69 , for division two 0.71, and for division three 0.67. The DSC between the images of the three divisions is below 0.56 .

The landmarks were placed manually, and $33+33$ landmark points were used to describe the right and left atrial blood mass shape. More 33 landmark points were used to define the cardiac boundary, which has the advantage that the initial model placing can be done more precisely. 
Blood Mass Extraction Algorithm: Initially a few parameters need to be set. For the ventricles the apex cordis and the base of the heart needs to be marked and for the atrial blood mass segmentation the three divisions of appearance have to be marked in the image data. The segmentation approach can be described in pseudo code the following way:

1. meanModel $=$ get mean model and calculate position of the model, where it has to be inserted initially

2. forall slides do begin

3. insert meanModel into the actual image slide

4. AAM initialization of the mean model:

- find the blood masses without adopting the model the best possible way.

5. solve minimization problem; iteration until convergence (AAM fitting)

6. meanModel $=$ model found during fitting procedure

- (this enhances the segmentation speed and prevents from missplacing during AAM initialization, because the adjacent image is (usually) similar to the just segmented one)

\section{7. end forall}

Figure 1 shows the different steps of the AAM model fitting approach. In this sample the convergence was reached after 15 iterations.
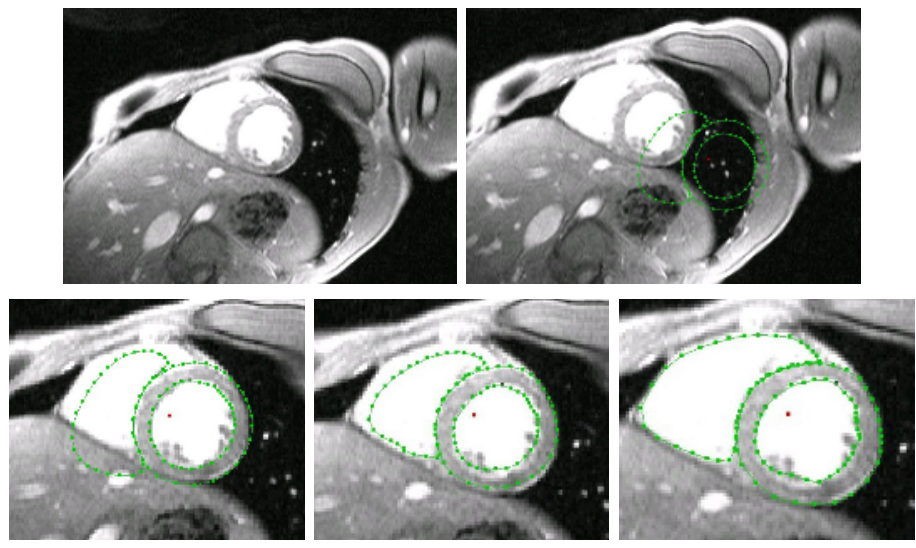

Fig. 1. Visualization of the AAM fitting approach. The shape models are superimposed to the medical images. The first image shows the medical image which is the input of the AAM segmentation task. The next slide shows the inserted mean model. The three images in the second row show the fitting process of the AAM search until convergence.

Myocardium Reconstruction: The myocardium reconstruction is based on a-priori knowledge provided by the atrial and ventricular blood masses. This approach employs mathematical and gray-value morphology operators [13. It uses virtual circles as structuring elements with a radius range from a defined minimum up to a maximum wall thickness. The structuring element rolls 
around the blood mass boundary and reconstructs the myocardial structure. If a voxel of the structuring element belongs to a gray-value range $g(V(x, y, z)) \in$ [minGray, $\max G r a y]$, then the voxel is part of the myocardium. The above described case occurs when the myocardium can be detected in the volume data, but especially when reconstructing the atrial myocardium it happens that the myocardium can not be detected in the volume data by the algorithm. In this case anatomical mean model information is used to reconstruct or estimate the myocardial structure. As standard parameter $4 \mathrm{~mm}$ are used for the atria and $8-10 \mathrm{~mm}$ for the ventricles. More information can be found in [14].

Assembling the VCM: After having all relevant compartments segmented, it is necessary to generate the VCM. Therefore, all segmented labelsets were triangulated using the standard marching cubes algorithm amiraDev ${ }^{\mathrm{TM}}$ provides. Because of the reason that the triangulation produces a lot of triangles an inhouse developed amiraDev ${ }^{\mathrm{TM}}$ plug-in is applied for remeshing and smoothing the generated surfaces. This process guarantees quality standards needed for solving the electrocardiographic inverse problem.

\section{Results}

Figure 2 shows three different patient individual VCMs. The left figure in row one shows a male, the right figure a female patient, where all compartments were extracted using the pipeline. The time for the extraction was about twelve minutes, and only a few manual corrections (atrial myocardium) had to be done. The second row shows a VCM of one patient in two different perspectives, without the compartment atria.

For testing the runtime behavior a $3.2 \mathrm{Ghz} \mathrm{CPU}$ with $1 \mathrm{~GB}$ of main memory was used. Overall, in this procedure, ten patient data (different volume data compared to the AAM training sets) with structural normal hearts were taken to generate the VCMs. All patients underwent a electrophysiology study (EPS) because they suffered from Wolff-Parkinson-White syndrome. CARTO ${ }^{\mathrm{TM}}$ maps, which are the gold-standard, were taken during the EPS study and compared with our model and our simulation results.

For the ventricular blood mass models one AAM was generated while the more complex geometry of the atria required to introduce three divisions of appearance and therefore, three AAMs were generated. This approach advanced the segmentation process for the atria.

The myocardium reconstruction is based of a-priori knowledge which is provided by the blood mass extraction method. In case the myocardium can be detected it can be reconstructed exactly, otherwise anatomical knowledge is used for reconstructing. This occurs predominantly when extracting the atrial myocardium.

The VCM integration was done by using the amiraDev ${ }^{\mathrm{TM}}$ tool with in-house developed extensions. 

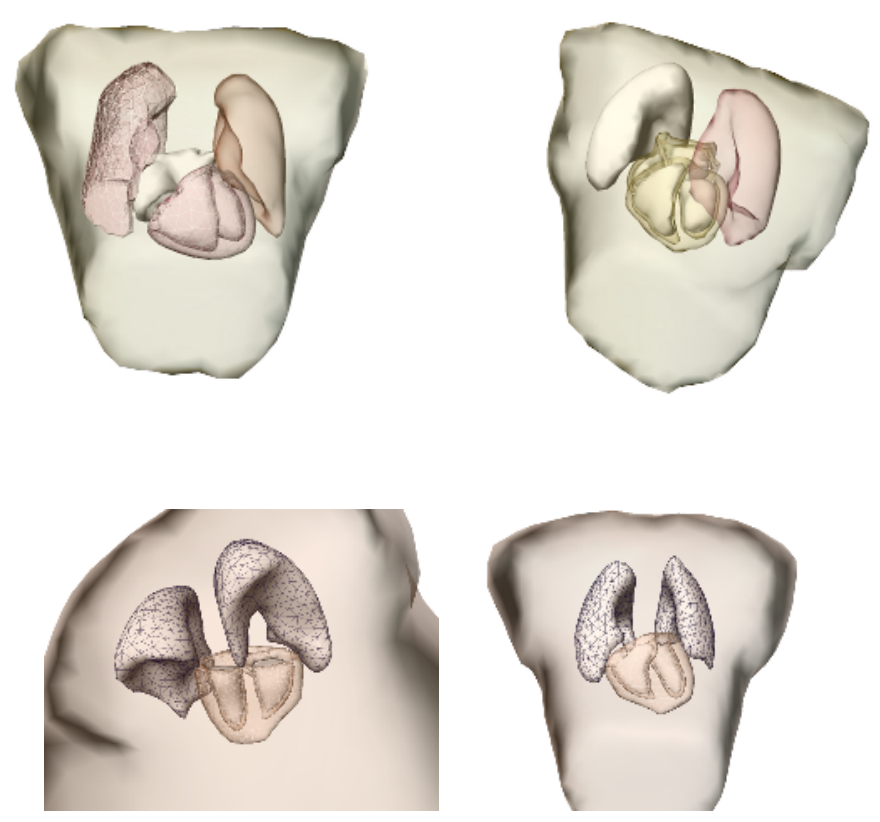

Fig. 2. Patient individual volume conductor models in different perspectives, visualized using amira ${ }^{\mathrm{TM}}$. For more details see text.

\section{Discussion}

The paper describes how a VCM can be extracted from morphological image data that qualifies for solving the electrocardiographic inverse problem.

One still existing problem is where the afferent blood masses (superior / inferior vena cava, pulmonary veins) should be separated. In our approach the afferent blood masses were separated as close as possible to the main target (the atria). This approach is supported by the fact that arrhythmias usually occur on the ventricle or atria and the veins have no or less electrical conductivity.

Furthermore, a major advantage of the proposed segmentation pipeline is, that only a few initial parameters have to be set and the overall runtime behavior is excellent. The generated models are of high quality and the model error is small, which is of importance when trying to solve the cardiographic inverse problem.

Because of the easy handling of the proposed approach, the good quality of the models and the good performance, this approach qualifies to be clinical applicable.

\section{Acknowledgements}

This study was supported by the Austrian Science Fund (FWF), grant STARTY144-N04. 


\section{References}

1. Gulrajani, R.: Models of the electrical activity of the heart and computer simulation of the electrocardiogram. Crit. Rev. Biomed. Eng. 16(1) (1988) p 1-66

2. Johnson, C.: Computational and numerical methods for bioelectric field problems. Crit. Rev. Biomed. Eng. 25(1) (1997) p 1-81

3. Ramanathan, C., Ghanem, R., Jia, P., Ryu, K., Rudy, Y.: Noninvasive electrocardiographic imaging for cardiac electrophysiology and arrhythmia. Nat Med. 10(4) (2004) p 422-8

4. Modre, R., Tilg, B., Fischer, G., Hanser, F., Messnarz, B., Schocke, M.S.M., Berger, T., Hintringer, F., Roithinger, F.: Atrial noninvasive activation mapping of paced rhythm data. J Cardiovasc Electrophysiol. 2003 Jul;14(7):720-1. 14(7) (2003) p $720-9$

5. van Rijn, R.A.C., Peper, A., Grimbergen, C.A.: High quality recording of bioelectric events. part 1 interference reduction, theory and practice. Med. Biol. Eng. Comput. 28(5) (1990) p 389-397

6. van Rijn, R.A.C., Peper, A., Grimbergen, C.A.: Highquality recording of bioelectric events. part 2. low-noise, low-power multichannel amplifier design. Med. Biol. Eng. Comput. 29(4) (1991) p 433-440

7. Andrea, E., Atie, J., Maciel, W., Araujo, N., Saad, E., Camanho, L., Affonso, H., Siqueira, L., Belo, L.G.: Mapping of supraventricular tachycardias by using a new three-dimensional technology: the carto system. J. Electrocardiol. 34(4) (2001) p 334-334

8. Bradley, C., Pullan, A., Hunter, P.: Effects of material properties and geometry on electrocardiographic forward simulations. Annals of Biomedical Engineering 28(7) (2000) p 721-741

9. Roberts, L.: Machine perception of 3-d solids. Optical and Electro-optical Information Processing. MIT press (1965) p 159-97

10. Heuberger, J., Geissbuehler, A., Mueller, H.: Lung CT segmentation for image retrieval. IPMI (2005)

11. Cootes, T., Taylor, C.: Active appearance models. 5th European Conference on Computer Vision; H. Burkhardt and B. Neumann (Springer 1998) p 484-498

12. Cootes, T., Taylor, C.: Active appearance models. http://www.isbe.man.ac.uk (2004)

13. Edward, R., Roberto, A.: Hands-on morphological image processing. SPIE Press, Bellingham, Washington USA (2003)

14. Pfeifer, B., Fischer, G., Hanser, F., Seger, M., Hintermueller, C., Modre-Osprian, R., Trieb, T., Tilg, B.: Atrial and ventricular myocardium extraction using modelbased techniques. Methods Inf. Med. 45 (2006) p 19-26 